\title{
CELL DETERMINATION AND DIFFERENTIATION OF IDENTIFIED SEROTONIN-IMMUNOREACTIVE NEURONS IN THE GRASSHOPPER EMBRYO ${ }^{1}$
}

\author{
PAUL H. TAGHERT ${ }^{2}$ AND COREY S. GOODMAN \\ Department of Biological Sciences, Stanford University, Stanford, California 94305
}

Received July 20, 1983; Revised October 3, 1983; Accepted October 3, 1983

\begin{abstract}
We have begun to investigate the factors that underlie neurotransmitter determination in the central nervous system of the grasshopper embryo. The most prominent serotonin-immunoreactive neurons in the segmental ganglia are three clonally related interneurons, cells S1, S2, and S3. S1 and S2 are sibling neurons and are the first two born in the family of neurons that is produced by neuroblast 7-3; cell S3 derives from one of the second-born pair in the same family. S1 is serotoninimmunoreactive in all thoracic and abdominal segments, $\mathrm{S} 2$ in all but two segments (T3 and A1), and S3 in only the prothorax (T1). These segment-specific differences are not due to differential cell death but rather can be ascribed to biochemical differences between lineally homologous neurons. Furthermore, these homologous neurons also display interesting segment-specific differences in their morphology. Laser ablation of neuroblast 7-3 before it begins its series of programmed cell divisions results in the absence of its normal serotonin-immunoreactive neuronal progeny when screened at mature stages. These experiments support the hypothesis that transmitter determination is at least in part regulated by cell lineage in the grasshopper embryo and that some clonally related neurons appear to share a common transmitter determination by virtue of their common lineage. The results further suggest that cell lineage regulation of transmitter determination may vary according to segment or may be modified by as yet unidentified segment-specific factors.
\end{abstract}

The differentiated nervous system displays a remarkable diversity of cell shape, chemistry, and function. For the most part, this diversity is generated during embryonic development, when, as a function of their mitotic ancestry and interactions with their environment, cells acquire specific instructions that inform (or restrict) their future fate. We are interested in the nature of these instructions and have focused our attention on those that restrict individual neurons to make particular transmitter substances.

In the neurogenesis of each segmental ganglion in the grasshopper, 61 stem cells, called neuroblasts (NBs), generate families of neuronal progeny that range in size from 6 to 100 neurons. In their study of the progeny of the median neuroblast (MNB), Goodman et al. (1980) presented evidence that most if not all of the neurons derived from the MNB produced octopamine as their neurotransmitter; included in this family is the identified

\footnotetext{
${ }^{1}$ We thank Joel Lupatkin for excellent technical assistance in this work. This research was supported by a Muscular Dystrophy Association Postdoctoral Fellowship to P. H. T. and by a grant from the National Institutes of Health to C. S. G.

${ }^{2}$ To whom correspondence should be addressed.
}

octopaminergic neuron DUMETI (Goodman and Spitzer, 1979). On this basis, Goodman et al. (1980) proposed the hypothesis that, in the grasshopper, the progeny from individual neuroblasts may share a common transmitter determination by virtue of their common ancestry. Can we demonstrate a correlation between cell lineage and cell fate for the progeny of other neuroblasts? Are entire neuroblast families, or subsets of these families, similarly instructed or restricted in their biochemical fate? Furthermore, can we demonstrate the validity of a cell lincage hypothesis of neurotransmitter determination by experimentally manipulating the normal patterns of cell divisions?

The central nervous system of the grasshopper embryo provides an attractive preparation in which to study the interaction between cell lineage and cell fate. The individual neurons and neuroblasts are large and identifiable at all developmental times and can be penetrated with intracellular microelectrodes (Goodman and Spitzer, 1979). Therefore, it is possible to follow and compare the anatomical, physiological, and biochemical differentiation of single identified neurons throughout the embryonic development of the central nervous system. In addition, descriptive cell lineages can be constructed, and, 
where this has been done, these lineages appear to be invariant (Goodman and Spitzer, 1979; Goodman and Bate, 1981; Raper et al., 1983). In the present study we first used immunocytochemical techniques and dye injections to identify and describe the differentiation of neurons that may contain serotonin in the central nervous system of the grasshopper. We then combined these techniques with cell ablations of the precursor cells that generate these and neighboring neurons. Some of the results have been previously reported (Taghert et al., 1982a).

\section{Materials and Methods}

We used embryos from a laboratory colony of Schislocerca americana for all experiments except those involving laser ablations. For these, we used embryos from a colony of a related species, Melanoplus differentialis, that were kindly provided by the Zoecon Corporation of Palo Alto, CA. All results concerning lineage, morphology, and segmental differences reported here were identical for both species.

Eggs of young embryos $(<55 \%$; see the time table of Bentley et al., 1979) were immersed in saline, cut open at their posterior end, and the embryo gently squeezed out. Embryos were mounted onto glass slides within "coffins" that were cut out of a layer of polymerized Sylgard (Corning) with the dorsal side up and fastened with metal pins that were secured beneath the Sylgard. Embryos older than $55 \%$ were further dissected by carefully removing the appendages, slitting the embryo along its dorsal margin, removing the gut, and then pinning the preparation ventral side up in a Sylgard-bottomed dish. The overlying epidermis was removed, and the ventral nerve cord was isolated and secured onto a glass coffin slide as described above. To aid visibility, the saline used was slightly hypotonic and consisted of 150 $\mathrm{mM} \mathrm{NaCl}, 3 \mathrm{mM} \mathrm{KCl}, 2 \mathrm{mM} \mathrm{CaCl}_{2}, 1 \mathrm{mM} \mathrm{MgSO}_{4}$, and 5 mM TES adjusted to $\mathrm{pH}$ 7.0.

Neurons were viewed using a Zeiss compound microscope fitted with Nomarski interference contrast optics and a Leitz 50X water immersion lens. Details of cell impalement and Lucifer Yellow (LY) dye injection techniques have been previously described (Raper et al., 1983). Following dye injection, preparations were fixed for $1 \mathrm{hr}$ in $2 \%$ paraformaldehyde in a Millonig's buffer $\left(0.1 \mathrm{M}\right.$ phosphate buffer with $0.001 \% \mathrm{CaCl}_{2}$ and $1.2 \%$ glucose, $\mathrm{pH}$ 7.3). Fixed dye-injected tissues were processed with a rabbit anti-Lucifer Yellow antiserum as previously described (Taghert et al., 1982b). Briefly, tissues were incubated with anti-LY antiserum overnight and then with an HRP-labeled goat anti-rabbit IgG (Miles). Following development of the HRP reaction product, tissues were rinsed in buffer, mounted in glycerin, viewed with Nomarski optics, and photographed and drawn at $\times 1250$ magnification.

For serotonin immunostaining, the isolated embryonic nervous system was fixed as described above and then processed according to the peroxidase-antiperoxidase (PAP) technique of Sternberger (1978). The fixed tissue was secured and incubated with antibodies within a glass coffin slide. Tissues were pre-incubated in $10 \%$ normal goat serum, $2 \%$ bovine serum albumin, and $1 \%$ Triton
$\mathrm{X}-100$ in phosphate-buffered saline (PBS) for $1 \mathrm{hr}$ at room. temperature. Tissues were then incubated in a rabbit anti-serotonin antiserum (Immunonuclear Corp.) at a 1:500 dilution, $1 \%$ normal goat serum, $1 \% \mathrm{BSA}$, and $1 \%$ Triton X-100 in PBS overnight at $4^{\circ} \mathrm{C}$. Following incubations in linking antibody and the PAP antibody complex for $1 \mathrm{hr}$ each at room temperature, the tissues were developed for the HRP reaction product, cleared, and examined as described above.

We used the grasshopper species $M$. differentialis for laser ablation experiments. These embryos were better suited for this purpose than were Schistocerca because the young (i.e., 30\%) Melanoplus embryos generally lie above the yolk mass and have fewer yolk granules between them and the egg shell. Hence, the neuroblasts in the neuroepithelial sheet were more clearly visible and more readily identifiable in ovo. Embryos of the proper developmental stage were dechorionated in bleach for approximately $30 \mathrm{sec}$ and then rinsed many times in saline. Single eggs were placed in Sylgard-coffin slides, immersed in a drop of saline, and covered with a coverslip that was itself covered with a drop of water. Gentle pressure on the coverslip caused a slight flattening of the egg and often improved visibility of the neuroblasts.

Neuroblasts were viewed under bright field conditions with a Zeiss compound microscope using a Leitz $50 \times$ water immersion lens. Laser ablations were performed with a Chromaser pumped-dye laser system (Phase-R Corp.) using Coumarin 504 dye (absorption maximum $\sim 500 \mathrm{~nm}$ ). Target cells were selected with a co-linear Helium-Neon gas laser; 5 to 10 shots at $\sim 250$ mjoules/ shot over 5 to $10 \mathrm{~min}$ were used to kill neuroblasts. Condensation of chromatin and subsequent loss of cellular integrity were seen within this time. Eggs were discarded given any sign of damage to neighboring, nontarget cells. Eggs with specific cellular ablations were removed from the slide, blotted dry, and then placed onto gauze that was moistened with antibiotic-containing saline (Penn/strep, Gibco). The eggs and gauze were stored in Petri dishes and incubated at $33^{\circ} \mathrm{C}$ until the embryos had reached either the $33 \%$ or $70 \%$ stage of embryonic development and then were studied as described under "Results."

\section{Results}

The neuroepithelium in each segment of the grasshopper embryo contains 61 neuroblasts (NBs; arranged in seven rows as shown in Fig. 10; Bate, 1976). These stem cells generate most of the neurons in each segmental ganglion (about 500 neurons in each abdominal ganglion and about 2000 neurons in each thoracic ganglion). Each NB can be uniquely identified by its position within the sheet, and some are now further identified according to their progeny: a stereotyped pattern of identified neurons that are generated by highly invariant cell lineages. NBs divide by a series of asymmetric divisions and give rise to ganglion mother cells; each of these smaller cells divides once more to produce two ganglion cells that differentiate into neurons. Individual NBs generate between 6 and 100 progeny.

The pattern of serotonin immunoreactivity. Immunocytochemical staining of the embryonic nervous system 

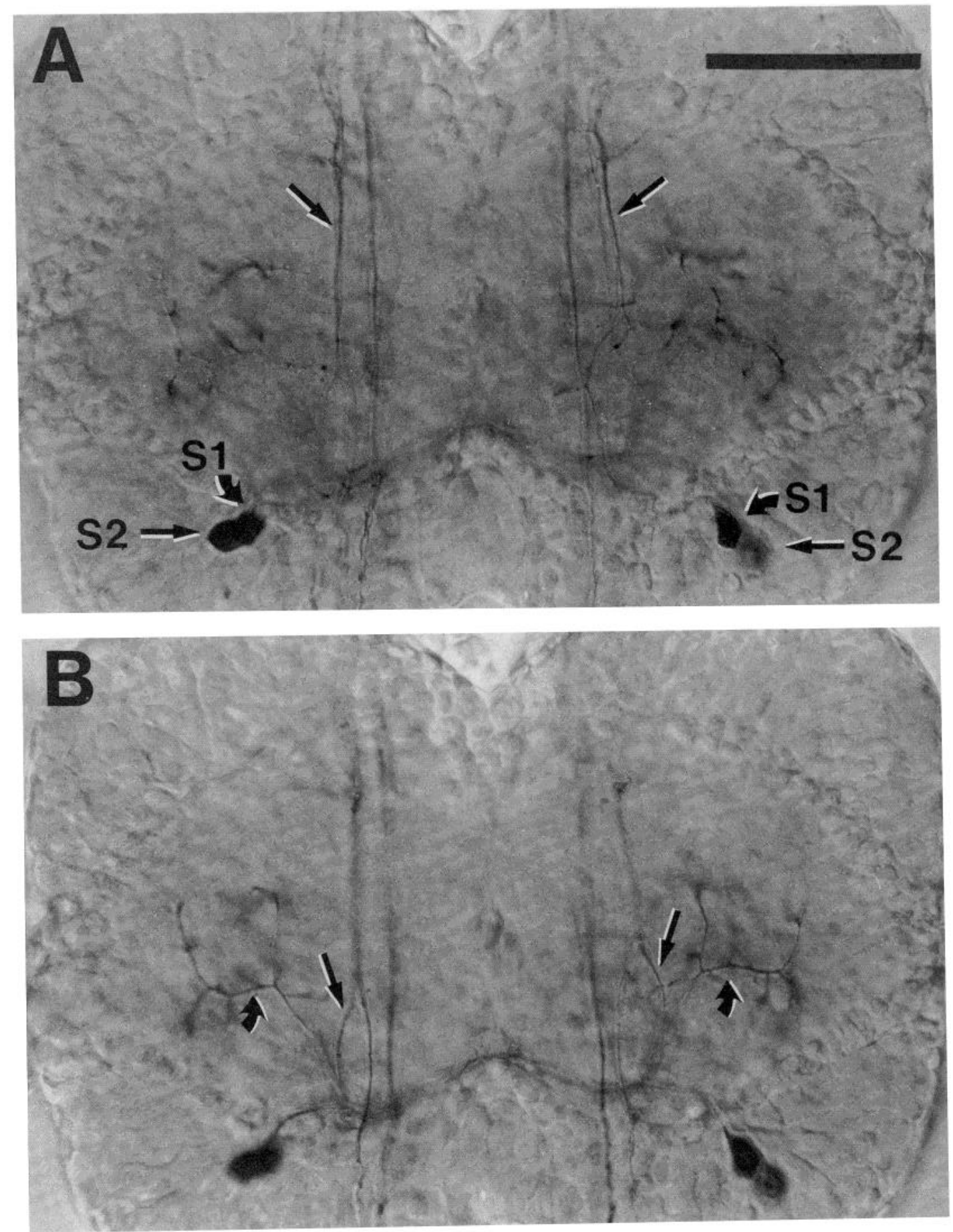

Figure 1. Photomicrograph of the mesothoracic ganglion (T2) from a $70 \%$ grasshopper embryo that was stained with the antiserotonin antiserum and processed with the peroxidase-antiperoxidase procedure in whole mount. $A$ and $B$ are two focal planes of the same tissue. Two pairs of immunoreactive cell bodies are seen: cells S1 and S2. Immunoreactive neuronal processes from S1 and S2 within this ganglion (arrows) and from homologous neurons in other ganglia are also seen. Calibration $=100$ $\mu \mathrm{m}$.

with the anti-serotonin antibody allows the complete visualization of the small number of immunoreactive (IR) neurons present in each ganglion (Fig. 1). Cell bodies, axons, and secondary branches are all clearly resolved. The number of neurons stained per segment is small (typically less than $1 \%$ of the neurons). The staining is specific to the extent that it is blocked by pre- incubation of the primary antiserum with serotonin $(100$ $\mu \mathrm{g} / \mathrm{ml}$ ) but not octopamine, dopamine, GABA, or acetylcholine. We have focused our attention on the most prominently staining IR neurons in each segment: these are the first cells that stain during embryonic development (see below), the most darkly staining, and the only ones to stain consistently. Their number varies strictly 


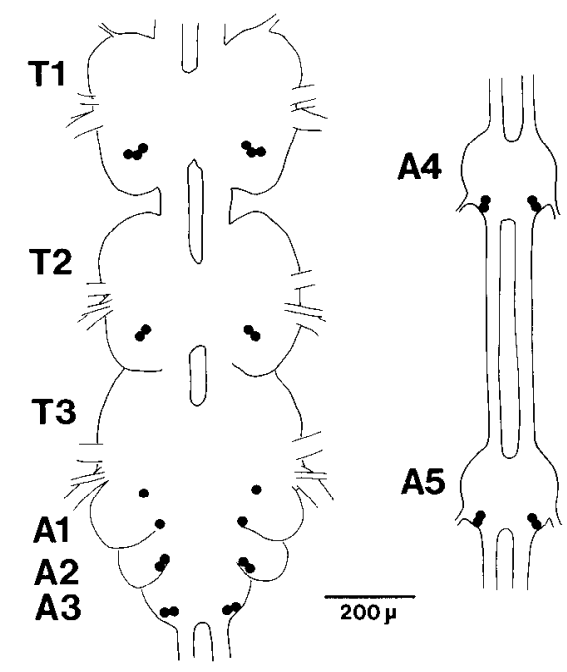

Figure 2. A schematic map of the most prominent immunoreactive cell bodies that are stained with the antiserotonin antiserum in thoracic and abdominal segments of a $100 \%$ grasshopper embryo. The segment-specific differences in immunoreactive cell bodies are seen consistently. Immunoreactive neurons are also present in the subesophageal, supraesophageal, and terminal abdominal ganglia but are not shown.

according to segment (Fig. 2): one IR cell is present on each side of the metathoracic (T3) and first abdominal ganglion (A1), two IR cells are present on each side of the mesothoracic (T2) and unfused abdominal ganglia (A4 to A6), and three immunoreactive cells are present on each side of the prothoracic (T1). This pattern is found in nearly mature embryos and is maintained throughout postembryonic life (data not shown). These cells thus identified according to their serotonin immunoreactivity and morphology (see below) are called the S1, S2, and S3 neurons. S1 stains in all segments; S2 stains in all segments but $\mathrm{T} 3$ and $\mathrm{A} 1 ; \mathrm{S} 3$ stains in segment T1 only.

Development of serotonin immunoreactivity. We were interested to determine when during embryonic development we could first detect serotonin IR. The embryonic CNS was stained in whole mount preparations every 3 to $4 \%$ from $40 \%$ to $60 \%$ of development (hatching occurs at $100 \%$; see Bentley et al., 1979). S1, S2, and S3 first exhibited IR between $54 \%$ and $56 \%$. An anterior to posterior gradient of staining onset was observed that was similar to the anterior-posterior developmental gradient previously noted in the timing of neuronal births and in many aspects of neuronal differentiation (Bate, 1976; Goodman and Bate, 1981). Staining was first observed in the thoracic segments and over the next $4 \%$ in nearly all abdominal segments. At this point in development, S1, S2, and S3 were postmitotic, had assumed their final cell body position relative to neighboring cells, and had already assumed their particular axonal morphologies (see below).

Individual immunoreactive neurons began to show complete staining at a particular point during the process of morphological differentiation. This is seen in the case of neuron $\mathrm{S} 1$ in thoracic ganglia stained between 55 and $65 \%$ of development (Fig. 3). In this segment, S1 becomes an intraganglionic interneuron (see below) whose sec-

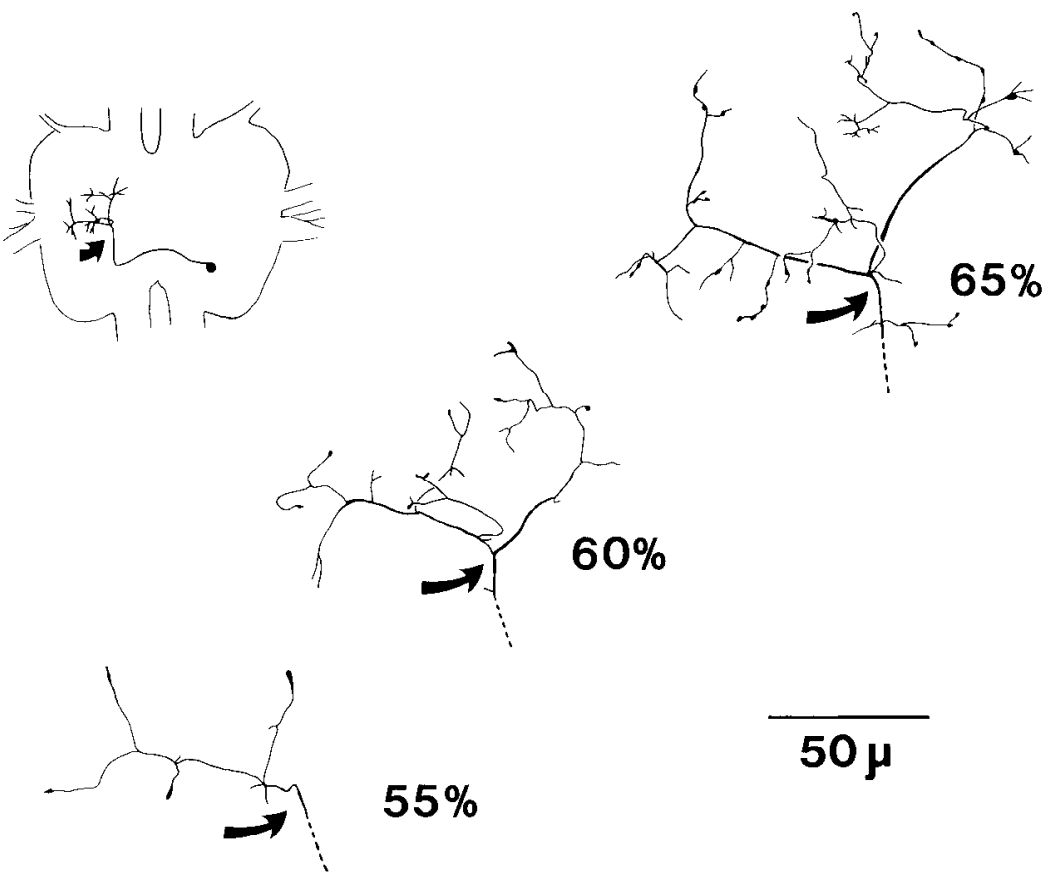

Figure 3. Embryonic neurons express the serotonin-like antigen before completing morphological development. Camera lucida drawings of the immunoreactive branches of cell $\mathrm{S} 1$ in the mesothoracic ganglion at three sequential times in development. Identified branches within the enlarging arbor contain the antigen throughout their length and within secondary growth cones. The arrow marks the major branch point in the arbor of cell $\mathrm{S} 1$; the inset (upper left) indicates the form of the mature neuron. 

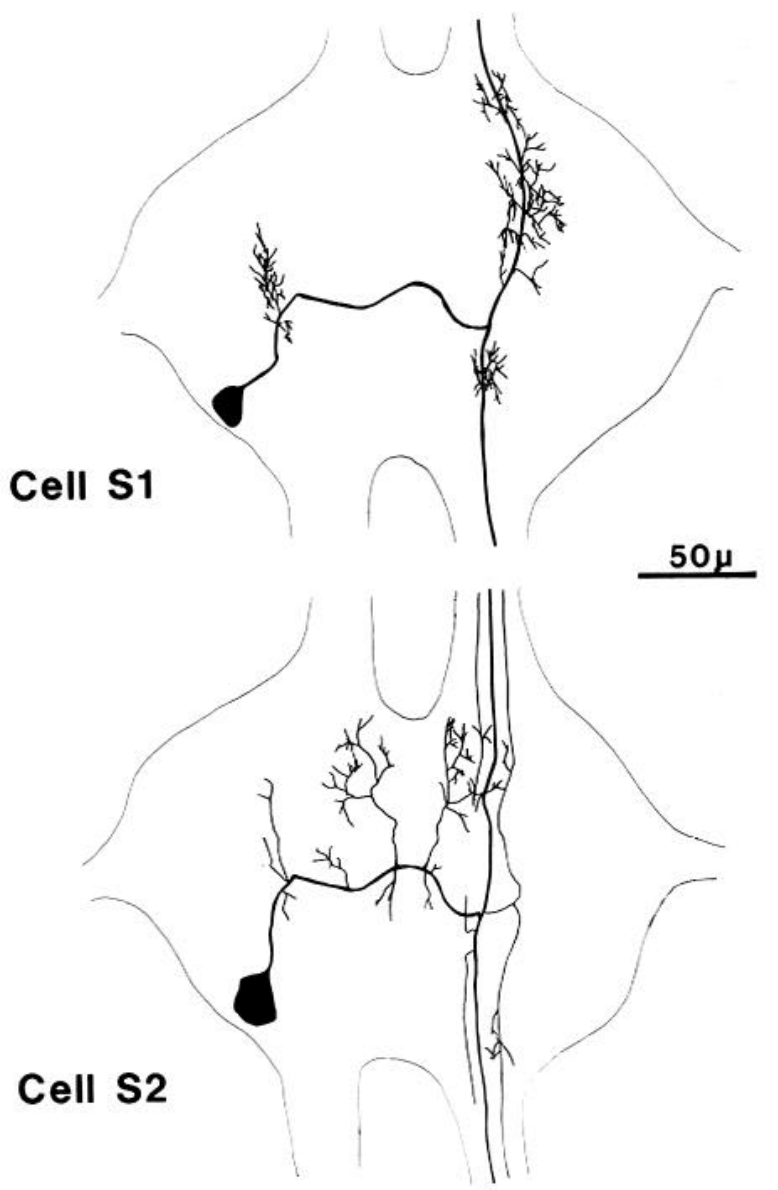

Figure 4. The morphology of two serotonin-immunoreactive neurons of abdominal ganglia at $70 \%$ of embryonic development as seen following Lucifer Yellow injection and anti-Lucifer Yellow antiserum treatment. Cells S1 and S2 are always serotonin-immunoreactive in segments $\mathrm{A} 4$ to $\mathrm{A} 6$. Both are interganglionic interneurons that project contralaterally in the same $\mathrm{C}$ commissure pathway. They are individually identifiable by the positions of their axons within the longitudinal connective and by their patterns of secondary branching.

ondary branches arborize on the side of the segment contralateral to the cell body. Figure 3 shows camera lucida drawings of immunoreactive branches of S1 at three, successively older stages and indicates the degree to which these branches accumulate the serotonin-like antigen while in the process of elaborating their final form. Reaction product was likewise present in the growth cones of immunoreactive interganglionic interneurons (data not shown). At each of these developmental stages, LY injections indicated that immunostaining with the anti-serotonin antiserum was revealing all the branches present. Thus, serotonin-like immunoreactivity appears before arborizations are fully elaborated. Furthermore, the antigen appears after growth cones have made their initial pathway choices but before they have reached their final targets.

Segment-specific morphology of identified neurons S1, $S 2$, and $S 3$. The cellular identity of cells $\mathrm{S} 1, \mathrm{~S} 2$, and $\mathrm{S} 3$ in segment $\mathrm{T} 1$, and $\mathrm{S} 1$ and $\mathrm{S} 2$ in segment $\mathrm{A} 4$ were ascertained by intracellular injections of LY into embryonic neurons in vivo at about $65 \%$ of embryonic devel- opment followed by reaction of the tissue with an antiLY antibody (Taghert et al., 1982b) to visualize better individual cell morphology. The same tissues were then processed for serotonin immunoreactivity to determine if $\mathrm{S} 1, \mathrm{~S} 2$, or $\mathrm{S} 3$ had been dye-filled. Based on the results of these experiments, the morphology of the two immunoreactive cells, $\mathrm{S} 1$ and $\mathrm{S} 2$ in the fourth abdominal ganglion (A4), is shown in Figure 4. Each neuron is an interganglionic interneuron that projects both anteriorly and posteriorly on the side of the nervous system contralateral to the cell bodies. Their axons cross the midline in the most ventral and most posterior axon bundle of the $\mathrm{C}$ commissure (Goodman and Bate, 1981). The axons of both cells (they often have multiple axons) project through at least two segments both anteriorly and posteriorly. The two cells are distinguished from one another by their particular patterns of secondary branching and by the stereotyped positions of their axons in particular axon bundles in the longitudinal connective: S1 axons are ventral and lateral, and S2 axons are more medial and dorsal. A third cell is frequently encountered in this area of A4 that has a similar morphology to that of S1 and S2 (Fig. 5). It crosses the C commissure in the same pathway but projects its axons in distinct, more ventral longitudinal axon pathways. Its central arborizations within A4 are symmetric and straight, and they remain ventral in the neuropil. This cell, S3, was encountered as frequently as was $\mathrm{S} 1$ or $\mathrm{S} 2$ but was not immunoreactive in segments A4 to A6 $(n=6)$. The reason for calling this cell S3 is based on its lineage as described below.

Much of the axonal morphology of the three immunoreactive cells in $\mathrm{T} 1$ could be ascertained with the antibody technique (c.f., Fig. 1). These details were confirmed by the dye injection of individual cells. Two of the cells are ascending interganglionic interneurons, and one is intraganglionic (Fig. 6). All three cross the midline in the most ventral and posterior axon bundle in the $\mathrm{C}$ commissure. Anteriorly, the two ascending interneurons project up into the brain. The immunoreactive cells in thoracic segments lack the distinctive central midline arborizations of cells in abdominal segments. The axonal

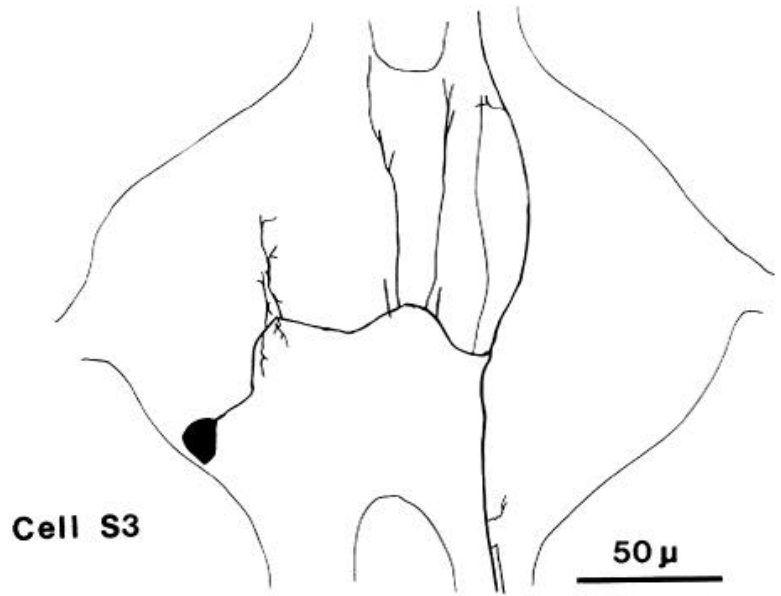

Figure 5. The morphology of a third cell, S3, that was frequently encountered in abdominal ganglia though was never serotonin-immunoreactive. Its cell body was adjacent to those of $\mathrm{S} 1$ and $\mathrm{S} 2$ and it crossed the midline of the ganglion in the same commissural axon bundle. 


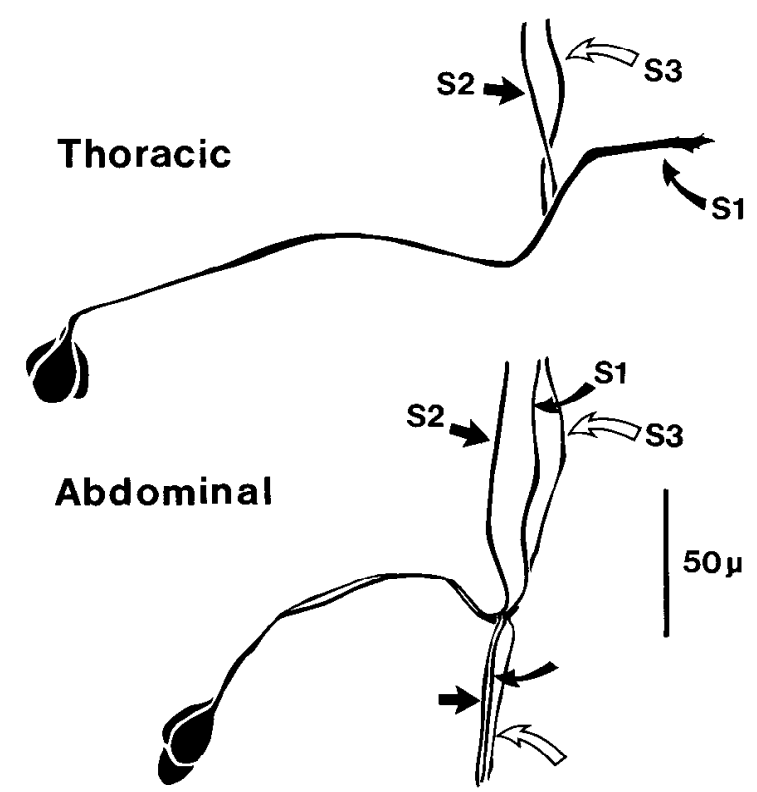

Figure 6. Cells S1, S2, and S3 display segment-specific axonal morphology. Camera lucida drawings of the basic axonal form of the three cells in thoracic ( $\mathrm{T} 1$-above) versus abdominal (A4-below) segments at approximately $60 \%$ of development. In all segments, the cells cross the midline in the same $\mathrm{C}$ commissure pathway, but, once on the other side, they grow on segment-specific pathways. Homology of neurons between segments is based upon morphological features and upon lineal position (see the text).

morphology of the immunoreactive neurons in $\mathrm{T} 1$ versus that seen in segment A4 is schematized in Figure 6. Because the immunoreactive cells in the different segments derive from the same neuroblast (see below), we have used the same nomenclature to identify them. S1 is the intraganglionic cell, $\mathrm{S} 2$ is the interganglionic cell whose axon loops dorsally and medially as it joins a longitudinally ascending pathway, and $\mathrm{S} 3$ is the interganglionic cell whose axon remains ventral as it exits the ganglion of origin.

Given their morphological identification, S1, S2, and S3 from embryos at successively earlier stages of development were dye filled in order to describe their pattern of growth (Figs. 7 and 8). As was shown for the growth of other grasshopper neurons (Goodman and Spitzer, 1979; Raper et al., 1983), cells S1, S2, and S3 follow an orderly pattern of morphological differentiation. In A4 (Fig. 7), the growth cone of S1 precedes that of S2 across the $\mathrm{C}$ commissure and in choosing its cell-specific longitudinal pathway; likewise, $\mathrm{S} 2$ always precedes $\mathrm{S} 3$ (data not shown). The elaboration of secondary branches begins following the establishment of the basic axonal form. In relation to their morphology, the cells first express immunoreactivity following the extension of axons onto specific longitudinal axonal pathways in the connective to neighboring segments and roughly coincident with the initiation of secondary branching. Despite the differences in final cell morphology, the same order of growth across the $\mathrm{C}$ commissure and onto cell-specific axon pathways was observed for the growth cones of cells S1, S2, and $\mathrm{S} 3$ in thoracic segments as well (Fig. 8). In all segments, this trio of cells was consistently dye-coupled (LY passed freely between them) up through approximately $50 \%$ of embryonic development.

Differences in the final morphology of segmentally homologous neurons can be a consequence of subtle differences in the choices made by their growth cones during axonogenesis. Consider the case of neuron $\mathrm{S} 1$, which is interganglionic in abdominal segments and intraganglionic in thoracic segments. The initial position of the cell body, the commissural pathway taken by its growth cone across the midline, and the contralateral longitudinal axon pathway upon which the growth cone extends all appear to be similar in each segment. It is only once the $\mathrm{S} 1$ growth cone has gotten off the commissural pathway and onto its cell-specific longitudinal pathway that differences are first apparent. In thoracic segments, the growth cone extends in the anterior direction exclusively, whereas in abdominal segments it bifurcates to extend both posteriorly and anteriorly. In addition, the thoracic S1 growth cone soon makes a second pathway alteration that directs it laterally into the neu-

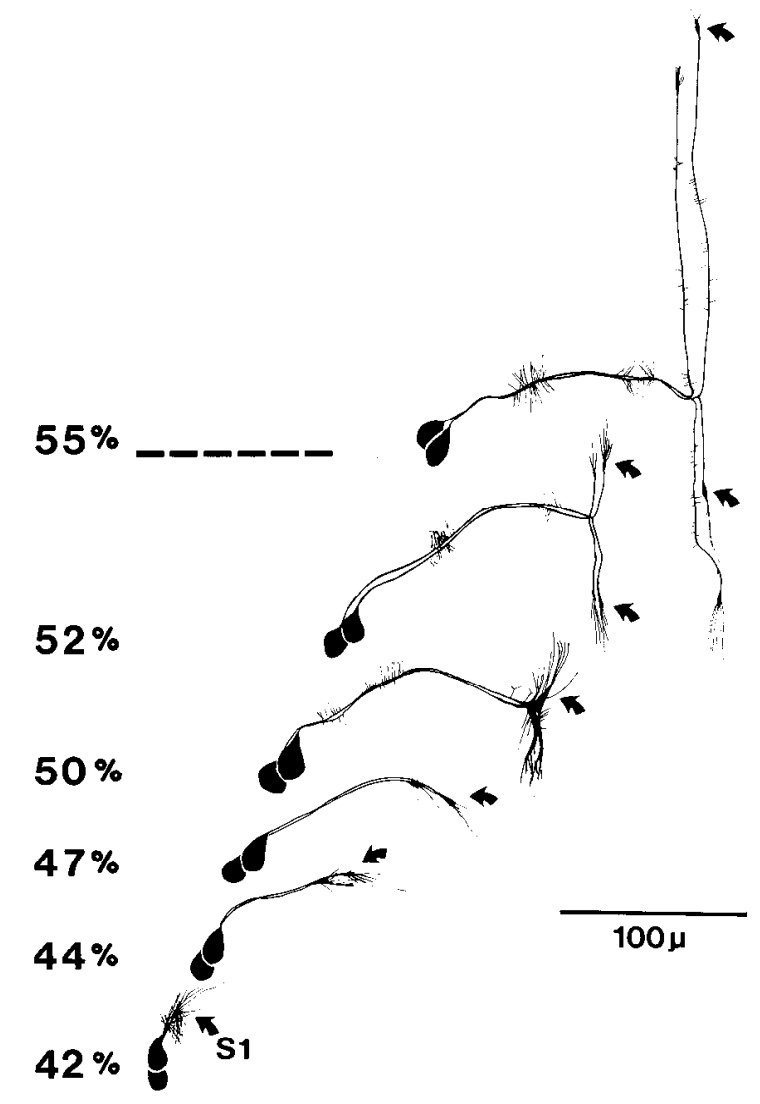

Figure 7. Elaboration of axonal morphology in cells $\mathrm{S} 1$ and $\mathrm{S} 2$ in the fourth abdominal segments at various, indicated times in embryonic development. At each time point in this series, the growth cone of cell S1 was slightly ahead of the S2 growth cone. Both cells become immunoreactive at about 55 to $58 \%$ of development (dashed line). At this time, both have axons that have assumed cell-specific positions in the longitudinal connectives and that have spanned approximately three segments. The growth cone of S3 (data not shown) follows that of $\mathrm{S} 2$ across the $\mathrm{C}$ commissure. The drawings are camera lucida representations of neurons filled with Lucifer Yellow dye and treated with an anti-LY antiserum. 


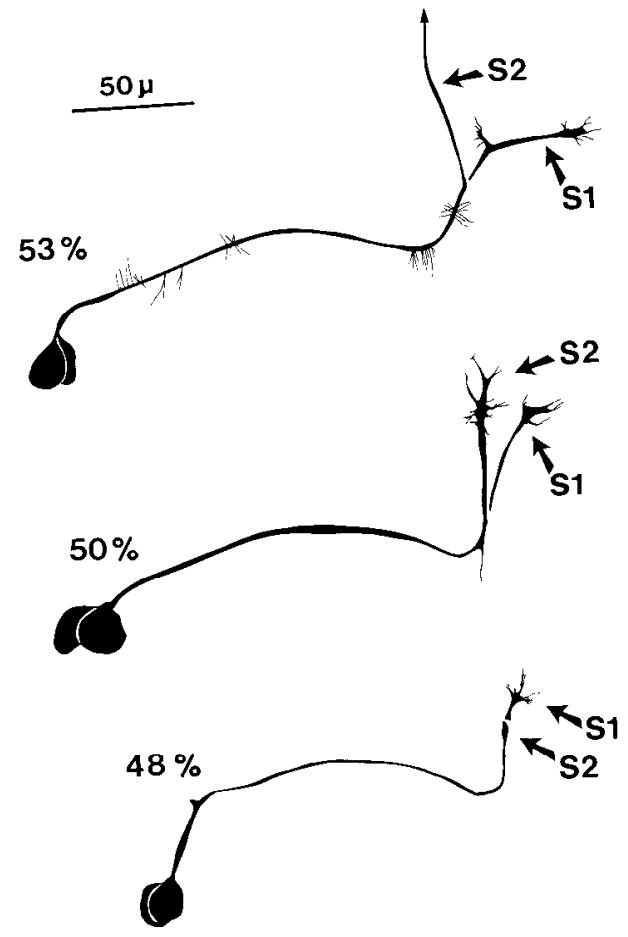

Figure 8. Axon outgrowth in cells $\mathrm{S} 1, \mathrm{~S} 2$, and $\mathrm{S} 3$ follows the same temporal order in thoracic as in abdominal ganglia. Camera lucida drawings of Lucifer Yellow-filled cells at three sequentially older times. The growth cone of cell S1 leads (followed by that of S2, then that of S3) and is the first to make its cell-specific turn off the C commissure pathway. In thoracic segments, $\mathrm{S} 1$ becomes an intraganglionic interneuron.

ropil of the segment of origin rather than into neighboring segments, as is the case in all abdominal segments.

Cell lineage of neurons $S 1, S 2$, and $S 3$. Having located the cell bodies of neurons $\mathrm{S} 1, \mathrm{~S} 2$, and $\mathrm{S} 3$ in older embryos and described their morphology by LY injection, we then reconstructed their lineage by examining younger embryos. We identified these cells in sequentially younger embryos on the basis of their characteristic morphology and cell body location. The cells were traced back to the stage at which they first extended their growth cones. At this early stage, cells $\mathrm{S} 1, \mathrm{~S} 2$, and $\mathrm{S} 3$ can be identified by the initial anteromedial trajectory of their growth cones onto a specific commissural pathway. Fortunately, at early stage, no other cell bodies in their region extend growth cones in that direction. By examining their location immediately adjacent to the younger ganglion mother cells from NB 7-3 family, we deduced that these three neurons were generated by NB 7-3. To test this lineage relationship directly, we took advantage of the fact that embryonic neurons remain dye-coupled (LY, $M_{\mathrm{r}}=450$ ) to their neuroblast of origin and sibling neurons through the time when they first extend growth cones (Goodman and Spitzer, 1979; Raper et al., 1983) (Fig. 9). We injected LY into cells S1, S2, or S3 at these early stages when they first initiate their growth cones, and we revealed the pattern of dye coupling to their family of origin by using the anti-LY antibody and HRP immunocytochemistry. From these preparations, we confirmed the finding that cells S1, S2, and S3 arise from NB 7-3. Moreover, LY injections of embryos at 3- to 4-

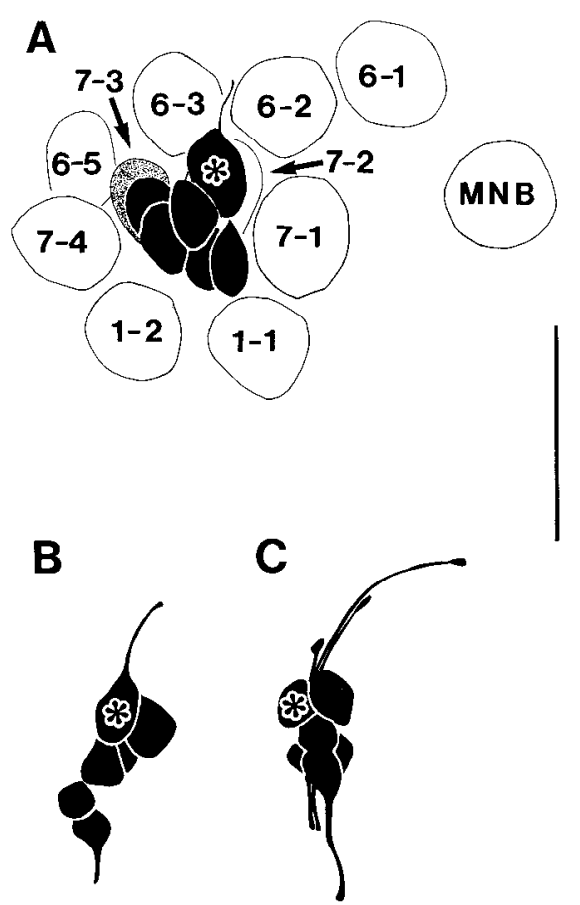

Figure 9. Cells S1, S2, and S3 are dye-coupled to their younger siblings and to NB 7-3 at the time when they first extend growth cones. Camera lucida drawings of anti-LYtreated embryos following dye injection of cell S1 (asterisk) at three stages of development. $A$, Only $\mathrm{S} 1$ has sent out filopodia toward the $\mathrm{C}$ commissure; dye can be traced back through five other neuronal progeny including S2 and S3 to NB 7-3 (stippled). Some of the other NBs in the vicinity of NB 7-3 are shown and numbered in open circles. $B$, At $2 \%$ later, the S1 growth cone is on the $\mathrm{C}$ commissure pathway; $\mathrm{S} 2$ and $\mathrm{S} 3$ have not yet extended growth cones, but a younger sibling has started to extend a growth cone posteriorly. By this time NB 7-3 has died. $C$, Approximately $2 \%$ later, the $\mathrm{S} 1$ growth cone is halfway across the $\mathrm{C}$ commissure and is followed by the $\mathrm{S} 2$ and $\mathrm{S} 3$ growth cones, in that order. The two youngest NB 7-3 progeny have started to extend growth cones posteriorly. Calibration: $A$, $58 \mu \mathrm{m} ; B$ and $C, 100 \mu \mathrm{m}$.

hr intervals of development allowed us to follow the birth order and growth cone initiation of these three neurons and thus assign their cell division of origin from NB 7-3 (Fig. 10).

These lineage assignments were rigorously established for segments T1 and A4, but this lineage appears similar in all other segments we have examined. We have followed cells $\mathrm{S} 1, \mathrm{~S} 2$, and $\mathrm{S} 3$ back into early embryos in segments T2, T3, A5, and A6 and have found their cell body location and initial growth cone extension to be identical to those in $\mathrm{T} 1$ and $\mathrm{A} 4$. In addition, less extensive lineage analysis in these other segments reveals the same NB 7-3 family and supports the interpretation that the NB 7-3 lineage we have described for segments T1 and A4 applies to these other segments as well.

NB 7-3 produces the smallest family of neurons in the segmental ganglia; it generates six neurons before undergoing programmed cell death. It is the first NB to die in each segment; it often produces a fourth ganglion mother cell that dies before dividing to produce neurons and at about the time the NB dies (C.M. Bate, personal 

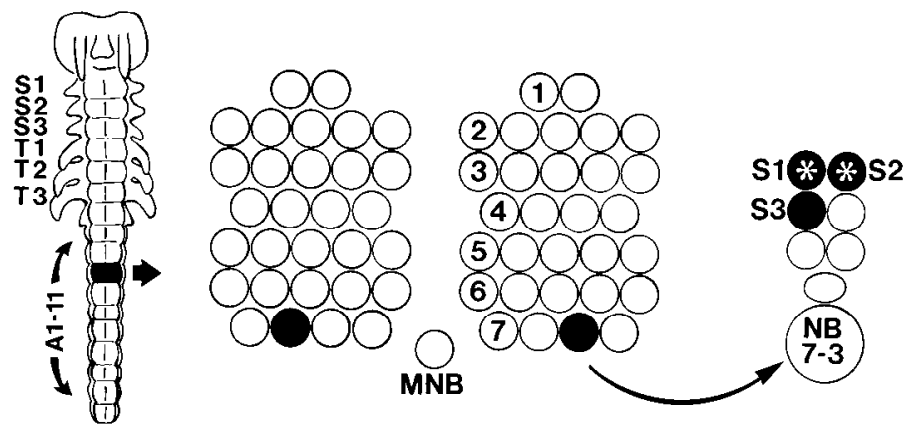

Figure 10. Cell lineage of the two serotonin-immunoreactive neurons in segment A4. The sheet of identified neuroblasts that generates each segmental ganglion is shown in the middle. Cells S1 and S2 (asterisks) are invariantly produced from the first division of NB 7-3, i.e., the third NB from the middle in the seventh row (solid circle). Cell S3 (also in black) is normally derived from the second pair of neurons, i.e., from the second cell division of NB 7-3. NB 7-3 sometimes produces a fourth ganglion mother cell that dies without producing neuronal progeny (C. M. Bate, personal communication).

communication). Cells $\mathrm{S} 1$ and $\mathrm{S} 2$ are the products of the first cell division of NB 7-3. S1 is the first to send out a growth cone, and it extends anteriorly and then curves medially toward the $\mathrm{C}$ commissure (Fig. 9, $A$ and $B$ ). S2 follows soon thereafter (Fig. $9 C$ ). Cell $\mathrm{S} 3$ is derived from the second cell division of NB 7-3, and its growth cone is extended following that of $\mathrm{S} 2$ (Fig. 9C). The $\mathrm{S} 3$ sibling and the two neurons that derive from the third ganglion mother cell of NB 7-3 project growth cones posteriorly and ventrally in a longitudinal axon bundle in the connective (Fig. 9C). The developmental fate of these three other neurons is not known.

Segment-specific transmitter expression. In addition to segment-specific differences in the axonal and secondary morphology of S1, S2, and S3, we have found segmentspecific differences in serotonin immunoreactivity of these lineally equivalent cells as well. Thus, $\mathrm{S} 1$ is strongly immunoreactive in all three thoracic segments and in all nine abdominal segments examined. S2 shows this property in all but segments $\mathrm{T} 3$ and $\mathrm{A} 1$; cell $\mathrm{S} 3$ is immunoreactive in $\mathrm{T} 1$ only. The absence of an immunoreactive neuron could be due to differential cell death among cells $\mathrm{S} 1$ to $\mathrm{S} 3$ in different segments. However, we know that this is not the case for at least one cell-S3; we routinely find this neuron with microelectrode dye injections in abdominal segments $\mathrm{A} 4$ to $\mathrm{A} 6$ in 60 to $70 \%$ embryos (this developmental time follows the period of massive embryonic cell death). Thus, lineally homologous neurons in the grasshopper CNS can be determined both morphologically and biochemically in a segment-specific manner.

The patterns of IR within the NB 7-3 family in different segments are summarized in Figure 11. These patterns are based on the study of more than 150 preparations and were found consistently in more than $95 \%$ of these. We do not know the fate (e.g., morphology or survival) of the three other neurons in the NB 7-3 family (the later siblings of $\mathrm{S} 1, \mathrm{~S} 2$, and $\mathrm{S} 3$ ). Therefore, we cannot speak as yet of the developmental fate of the entire NB 7-3 family. However, consideration of the fates of $\mathrm{S} 1$ to $\mathrm{S} 3$ does allow the following points to be made.

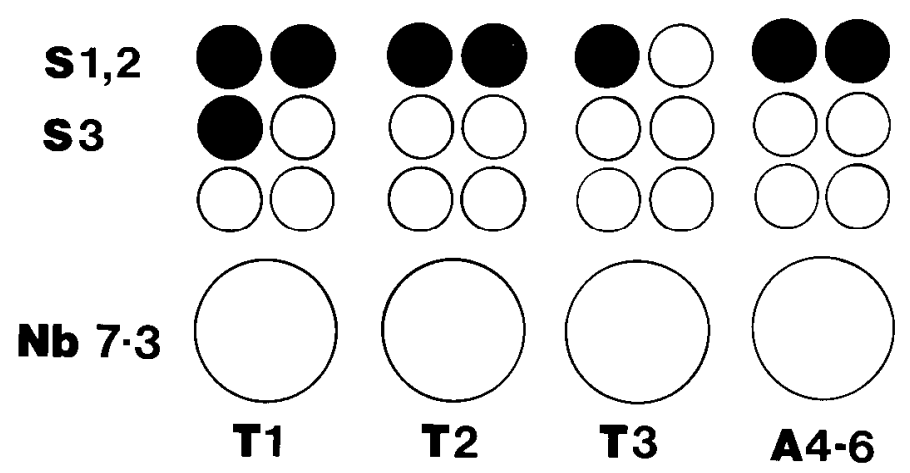

Figure 11. The pattern of serotonin immunoreactivity within the NB 7-3 family varies in a segment-specific fashion. The family is schematically diagrammed for segments $\mathrm{T} 1$ to $\mathrm{T} 3, \mathrm{~A} 1$, and A4 to A6. Cells S1, S2, and S3 survive in all segments illustrated. Solid circles indicate those progeny that become immunoreactive. Cell S1 is immunoreactive in all segments, S2 in all but segments $\mathrm{T} 3$ and $\mathrm{A} 1$, and $\mathrm{S} 3$ in segment $\mathrm{T} 1$ only. The fate (morphology or survival) of the three remaining neurons in this family is not presently known.

(i) Clonally related neurons tend to share neurotransmitter phenotypes. However, (ii) not all progeny of NB 7-3 share common levels of neurotransmitter immunoreactivity; e.g., in $\mathrm{A} 4$ to $\mathrm{A} 6, \mathrm{~S} 1, \mathrm{~S} 2$, and $\mathrm{S} 3$ all survive, yet only S1 and S2 show IR. (iii) Lineally equivalent neurons express serotonin IR as a function of their segment; cell S3 is immunoreactive in T1 but not in other segments. (iv) There is a gradient in the number of IR NB 7-3 neurons that declines from anterior to posterior through the thoracic segments and remains constant (at two) in abdominal segments.

Laser ablation of NB 7-3. Segment-specific differences in the IR within the NB 7-3 family indicate the possibility that the local cellular and/or noncellular environment might influence the transmitter determination of these neurons. Alternatively, their invariant cell lineage suggests that the determination or perhaps the competence to acquire it may derive from their mitotic ancestry. One way to test the latter hypothesis (one of cell lineage determination) would be to eliminate the lineage that normally generates $\mathrm{S} 1, \mathrm{~S} 2$, and $\mathrm{S} 3$ and to test later in development for the ability of other NBs to produce serotonin-immunoreactive neurons.

'Iherefore, we ablated NB 7-3 on one side in abdominal segments 4,5 , or 6 with a laser microbeam in ovo 1 to 2 hr before its first cell division. The contralateral NB 7-3 served as a control. In separate experiments, the neighboring NB 6-5 was similarly ablated. Some experimental embryos were dissected approximately $24 \mathrm{hr}$ following the ablation (at $33 \%$ of development) to test for the presence or absence of the NB. Interestingly, although we found that in approximately $80 \%$ of these cases $(n>$ 20) NB 7-3 was missing, in a few cases, the NB and progeny were present. This occasional failure to remove the neuroblast permanently is probably not due (i) to our inability initially to ablate the target NB, or (ii) to regulation between adjacent NBs. Rather, other experiments, described in more detail under "Discussion," demonstrate that NB replacement is due to regulation by neighboring epidermal cells; once an NB begins its first cell division, regulation no longer occurs (Doe and Good- 

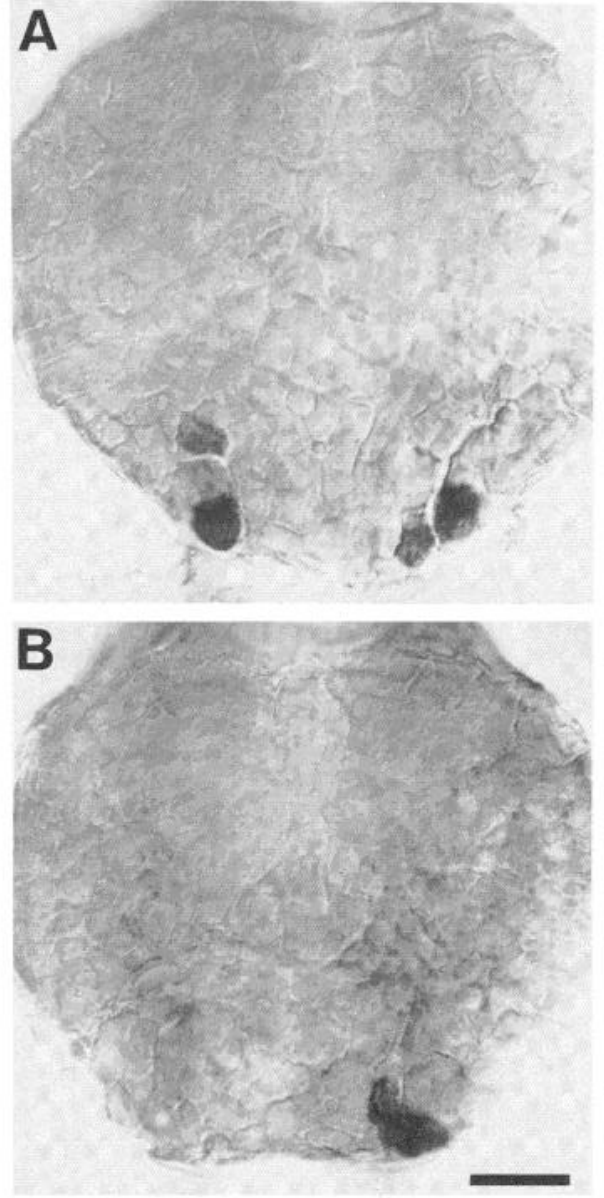

Figure 12. Laser ablation of NB 7-3 before it has begun its cycle of cell divisions leads to the absence of its identified progeny, S1 and S2 in segment A4. A, Photomicrograph of an unoperated control ganglion stained in whole mount with the antiserotonin antiserum at $70 \%$ of embryonic development. Note the bilaterally symmetric immunoreactive cells, S1 and S2. $B$, Photomicrograph of a similarly stained ganglion in which NB 7-3 had been laser ablated earlier in development. Note the absence of immunoreactive cells on the operated side. See the text for further details. Calibration $=30 \mu \mathrm{m}$.

man, 1983; Taghert et al., 1983). In most cases, when we laser ablate an NB just before it begins its first cell division, no such regulation from epidermal cells takes place.

Therefore, given the ability to ablate NB 7-3, it was possible to inquire as to the later disposition of the serotonin-immunoreactive neurons. Following growth to $70 \%$ of embryonic development, the CNS was isolated and processed for serotonin IR in whole mount. In 7 of 10 cases in which NB 7-3 had been ablated, no IR cells were present on the experimental side of the ganglion (Fig. 12). In these examples, the contralaterally homologous IR neurons were always present and had normal morphological appearances. In three cases, the normal pattern of two IR cells on both sides was observed. Thus, following NB ablation, serotonin-immunoreactive progeny were missing at a rate roughly equivalent to that at which we were able permanently to ablate the neuroblast in ovo. Ablation of NB 6-5 had no effect on the resultant pattern or morphology of IR cells $(n=20)$. In the majority of cases, therefore, the absence of the specific neuroblast 7-3 led to the absence of neurons that could express this specific determination. The progeny of neighboring NBs did not replace the absent NB 7-3 progeny.

Pattern duplication. The pattern of serotonin-IR cells in the grasshopper CNS provides a clear example of the precise and nearly invariant mechanisms that underlie the generation of cell diversity in the nervous system. In more than 150 immunostained nervous systems that were examined, S1, S2, and S3 were nearly always present in the proper position and stained in the segment-specific manner. Therefore, we were interested to observe a striking addition to the normal pattern in a single segment on a single occasion.

In this instance, a partial duplication had apparently occurred in the mesothoracic ganglion such that, in addition to the two immunoreactive cells S1, S2, and cell $\mathrm{S} 3$ present in the posterior half of the ganglion, three additional prominently stained neurons were present in the anterior half (Fig. 13). Their cell body position was roughly symmetric to that of the $\mathrm{S}$ neurons across the anterior-posterior axis of the ganglion. The axonal morphology of two of these cells could be resolved by virtue of antibody staining. Both crossed the midline in a very ventral and anterior pathway in the A commissure. One

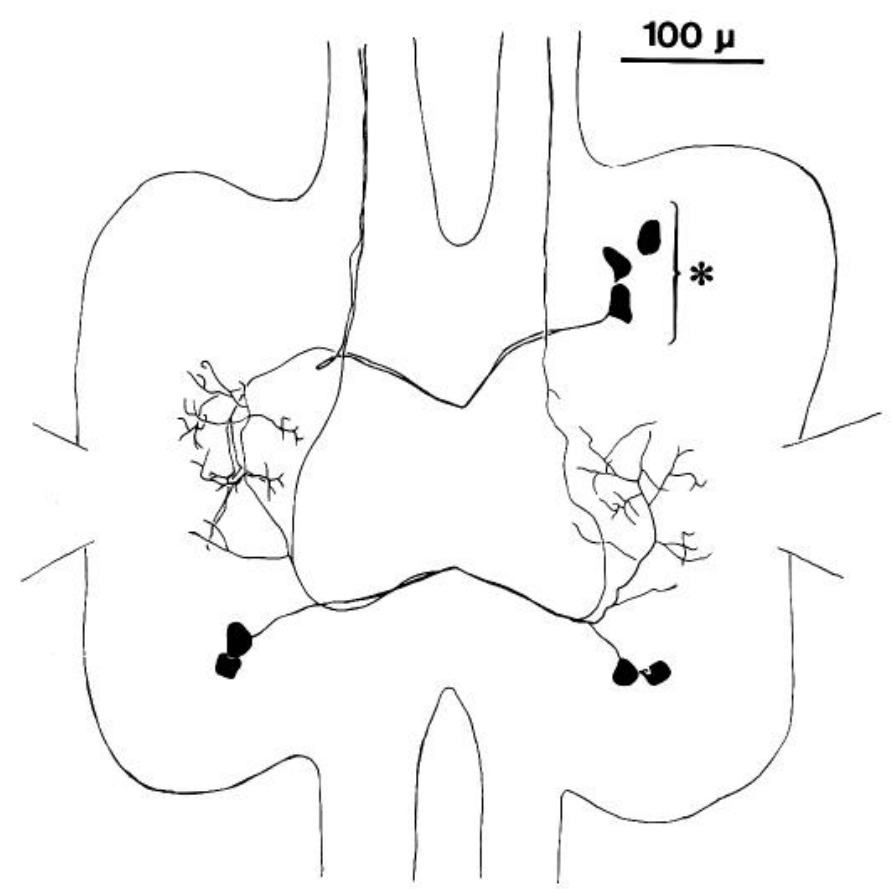

Figure 13. An unusual case of immunoreactive cell duplication. Normally, IR cells are present only in the posterior half of each ganglion. In this example, an extra set of neurons was stained in the anterior half. Camera lucida drawing of a whole mount mesothoracic ganglion from $M$. differentialis at $70 \%$ of embryonic development. The extra neurons (asterisk) display many symmetries with respect to the normally staining neurons; these include cell body position, commissural pathway, and axonal morphology in the two cells where this can be resolved. Note the overlap of branches in S1 and the "extra" intraganglion interneuron, the symmetry of commissural pathway, and the addition of a second extra immunoreactive axon onto that of the normal S2. 
cell was an intraganglionic interneuron and had branches that closely overlapped those of the intraganglionic neuron S1. The second additional cell looped dorsally onto the same anteriorly directed longitudinal pathway that was followed by cell S2. The morphology of the third cell could not be resolved. Hence, in this rare case of IR cell duplication, we note the following symmetries with respect to the anterior-posterior axis of the ganglion: (i) cell body position, (ii) commissural pathway, and (iii) cell-specific morphology including longitudinal axonal pathway. One asymmetric feature was evident: The "duplicated S2" observed the same posterior-to-anterior polarity of axon growth seen in the "normal S2."

\section{Discussion}

Immunocytochemical techniques applied to the nervous system of the grasshopper embryo can be a rapid and sensitive method for assessing the transmitter content of groups of identified neurons. The use of the antiserotonin antibody has indicated those neurons in the grasshopper CNS which are likely to use this biogenic amine; the following points support this possibility. (i) The antiserum is more sensitive to synthetic serotonin than it is to other putative neurotransmitters. (ii) The onset of serotonin immunoreactivity in the grasshopper CNS (at about $55 \%$ of embryonic development) compares favorably with developmental time at which serotonin can first be detected in this animal with a radioenzymatic assay (C. S. Goodman and R. McCaman, unpublished results). (iii) Similarly prepared antisera have been shown to stain known serotonergic neurons in the leech (J. Glover, personal communication), a serotinergic nerve plexus in the lobster nervous system (Beltz and Kravitz, 1983), and subpopulations of cells in areas of the rat brain (Kohler et al., 1982) and the mouse enteric nervous system (Rothman and Gershon, 1982) that are known to contain serotonin.

We have no method for estimating the sensitivity of the immunocytochemical technique and hence cannot quantify the minimum levels of detection. We have interpreted the presence versus the absence of serotonin immunoreactivity to indicate a qualitative difference in serotonin expression. It is possible that neurons that are not immunoreactive in our hands (e.g., cell S3 in all segments but T1) do produce an undetectable level. Nevertheless, the consistent and dramatic differences in levels of immunoreactivity that we do see clearly indicate differences in levels of expression that are segment- and cell-specific.

The orderly schedule of morphological and neurochemical differentiation displayed by monoaminergic neurons in grasshopper embryos parallels the results of studies of developing neurons in the vertebrate CNS. Cells S1, S2, and S3 begin to express their neurotransmitter phenotype after they become postmitotic and after they have initiated axonogenesis but before they have reached their final targets and before they have fully elaborated their complete morphology. Similarly, catecholaminergic neurons in the central nervous system of the rat begin to express tyrosine hydroxylase (TH) immunoreactivity following their withdrawal from the cell cycle but during the period of cell migration (Specht et al., 1981). These cells have elaborated growth cones that are TH-immunoreactive but that have not yet reached their final targets. Nor have these cells completed the construction of their terminal fields. In addition, there is evidence to suggest that neurons in the rat brain that contain substance $\mathrm{P}$ and others that contain met-enkephalin begin to express their transmitter phenotypes before they have completely elaborated their terminal fields (Pickel et al., 1982). This schedule suggests that, in these cases, the initial transmitter determination may precede any interactions the neurons may have with their target cells. It should be noted, however, that positive feedback interactions from postsynaptic targets have been shown to influence the biochemical maturation (i.e., the level of expression) of presynaptic neurons (Black et al., 1972).

The nature of factors that affect or alter initial neurotransmitter determination has been studied extensively in cells of the vertebrate peripheral nervous system (e.g. Black, 1978; Patterson, 1978). These studies have revealed plasticity in the ability of autonomic neurons that are derived from the neural crest to choose between different transmitter programs. Environmental influences during the course of migration from the neural crest to final target sites can reverse initial neurochemical differentiation from catecholaminergic to cholinergic expression in cell populations in vivo (LeDouarin et al., 1982) and in single cells in vitro (Patterson and Chun, 1977). Likewise, during postnatal development, sensory neurons also derived from neural crest cell can regulate levels of substance $\mathrm{P}$ and somatostatin under the influence of non-neuronal cells in vitro (Mudge, 1981).

We have not specifically examined the role that cellular interactions may play in the transmitter determination of cells S1, S2, and S3. However, the following observations make the possibility of certain interactions unlikely. As mentioned above, the early expression of the serotonin-like antigen argues against a significant instructive influence on the part of the targets of $\mathrm{S} 1, \mathrm{~S} 2$, or S3. Likewise, we cannot correlate any specific morphological features of these three neurons with serotonin immunoreactivity. For example, morphologically equivalent cells (S2 in segments T2 and T3) show different levels of immunoreactivity, whereas morphologically unequivalent homologues ( $\mathrm{S} 1$ in segments $\mathrm{T} 2$ and $\mathrm{A} 4$ ) show similar levels of expression. These results argue against the possibility that the biochemical determination of these neurons is causally related to their specific morphology.

One morphogenetic feature is positively correlated with serotonin immunoreactivity, and it involves age differences between cells S1, S2, and S3 in any given segment. Within this trio of neurons in all segments, S1 is the first to extend a growth cone, the first to navigate across the $\mathrm{C}$ commissure axon bundle, and the first to make its cell-specific pathway choice on the contralateral side of the ganglion. In this sense, we term cell S1 "older" than cell S2. S2 follows $\mathrm{S} 1$ and is in turn followed by the "younger" cell S3. In any segment in which not all three neurons are immunoreactive, then those which are immunoreactive are "older" than those which are not im- 
munoreactive. For example, in segment T2, one of the three neurons is not immunoreactive and it is the "youngest", cell S3; in segments T3 and A1, two neurons are not immunoreactive and they are S2 and S3. Whether this correlation represents either a change in some environmental influence that is sequentially encountered by the growth cones or cell bodies of these three cells or, alternatively, intrinsic segment-specific differences in these three cells remains to be demonstrated.

We have not excluded a role for extrinsic influences, but the experimental results lead us to favor a cell lineage model of transmitter determination. The three neurons that were identified with the antibody technique are all derived from the same neuroblast, NB 7-3, in an invariant fashion. The strongest evidence to support a lineage model comes from the neuroblast ablation experiment. Laser ablation of NB 7-3 just before it begins its programmed series of divisions results in the lack of its normal serotonin-immunoreactive progeny later in development. Two points can be made from this observation. The first concerns neuroblast determination and the second neuronal determination.

First, in the case of NB 7-3, the commitment to the NB 7-3 fate appears to be irrevocable near or at the time of its first cell division. Thus, in its absence, no other NB or neighboring epidermal cell is capable of generating the IR cells S1 or S2, i.e., no other cells are capable of assuming the NB 7-3 fate. Interestingly, laser ablation of individual epidermal cells that are the precursors to NB 7-3 results in the recruitment of a neighboring epidermal cell to assume that fate (Doe and Goodman, 1983). T'This regulative capacity of NB 7-3 precursors may explain our partial (70\%) success rate in eliminating this neuroblast. Thus, our interpretation is that in the case of NB 7-3, neuroblast determination appears to be regulative up to the time of its first cell division and cell autonomous thereafter (Taghert et al., 1983).

A second point to be made is that transmitter expression in the $\mathbf{S}$ neurons derives, at least in part, from the normally invariant NB 7-3 lineage. Some form of exclusive determination from NB 7-3 to its first few progeny, whether it be instructive or permissive, is indicated by the fact that no neighboring NBs or neurons could replace the experimentally induced deficit. The importance of cell lineage in the governance of transmitter phenotype has previously been demonstrated by similar laser ablation techniques in the nematode worm (Sulston and Horvitz, 1977).

Cells S1, S2, and S3 survive the period of embryonic cell death in segments $\mathrm{T} 1$ to $\mathrm{T} 3$ and $\mathrm{A} 4$ to $\mathrm{A} 6$, yet they express serotonin immunoreactivity differentially and in a segment-specific manner. Thus, while the neuroblast elimination experiment demonstrates the importance of ancestry for transmitter expression in these neurons, segment-specific expression indicates that the instructive process is complex and may include a second component. This component would dictate which among the NB 7-3 progeny in various segments do and which do not assume this transmitter phenotype. Therefore, we hypothesize that grasshopper neuroblasts confer to their specific progeny a common determination (or a competence to share a common determination) and that addi- tional, segment-specific factors influence the pattern of expression within neuroblast families. Whether this additional level of instruction involves cell ancestry (i.e., differences between equivalent NBs in various segments) or is shaped by segment-specific factors that are extrinsic to the 7-3 family remains a mystery, although a testable one.

If serotonin-immunoreactive neurons acquire their transmitter phenotype largely due to ancestry from NB $7-3$, then our question regarding the role of lineage in transmitter determination may be pushed back to an earlier developmental time. How is it that this specific NB acquires its special properties? We have little information concerning neuroblast determination in the insect neuroepithelium (see Bate, 1982; Taghert et al., 1982a). In this regard, however, the single example of duplication of serotonin-immunoreactive neurons is intriguing in that the two sets of neurons exhibit many mirror image symmetries with respect to the anteriorposterior axis of the ganglion. These symmetries include cell body position, cell-specific axonal morphologies, and secondary branching patterns. This observation suggests the possibility that embryonic ganglia may be divisible into quasi-symmetric morphogenetic fields. The demonstration of morphogenetic fields-termed compartments - has been used to describe pattern formation in the ectoderm in a variety of insects (e.g., Lawrence, 1981). Interestingly, the neuroepithelium arises from this same ectoderm. Whether similar processes of anterior and posterior compartmentation within a segment act on the developing neuroectoderm to help define neuroblast and neuronal cell fate remains to be demonstrated.

\section{References}

Bate, C. M. (1976) Embryogenesis of an insect nervous system. I. A map of the thoracic and abdominal neuroblasts in Locusta migratoria. J. Embryol. Exp. Morphol. 35: 107-123.

Bate, C. M. (1982) Proliferation and pattern formation in the embryonic nervous system of the grasshopper. Neurosci. Res. Program Bull. 20: 803-812.

Beltz, B. S., and E. A. Kravitz (1983) Mapping of serotoninlike immunoreactivity in the lobster nervous system. J. Neurosci. 3: 585-602.

Bentley, D., H. Keshishian, M. Shankland, and A. ToroianRaymond (1979) Quantitative staging of embryonic development of the grasshopper Schistocerca nitens. J. Embryol. Exp. Morphol. 61: 47-74.

Black, I. B. (1978) Regulation of autonomic development. Annu. Rev. Neurosci. 1: 183-214.

Black, I. B., I. A. Hendry, and L. L. Iverson (1972) The role of post-synaptic neurons in the biochemical maturation of presynaptic cholinergic nerve terminals in a mouse sympathetic ganglion. J. Physiol. (Lond.) 221: 149-159.

Doe, C. Q., and C. S. Goodman (1983) Regulation and determination of neuronal precursor cells revealed by cell ablations in the grasshopper embryo. Soc. Neurosci. Abstr. 9: 889.

Goodman, C. S., and C. M. Bate (1981) Neuronal development in the grasshopper. Trends Neurosci. 4: 163-169.

Goodman, C. S., and N. C. Spizer (1979) Embryonic development of identified neurones: Differentiation from neuroblast to neurone. Nature 286: 208-214.

Goodman, C. S., K. G. Pearson, and N. C. Spitzer (1980) A spectrum of properties in the progeny of a single neuronal 
stem cell. Proc. Natl. Acad. Sci. U. S. A. 77: 1676-1680.

Kohler, C., V. Chan-Palay, and H. Steinbusch (1982) The distribution and origin of serotonin-containing neurons in the septal area: A combined immunohistochemical and fluorescent retrograde tracing study in the rat. J. Comp. Neurol. 209: 91-111.

Lawrence, P. A. (1981) The cellular basis of segmentation in an insect. Cell 26: 3-10.

T.eDouarin N. M., J. Smith, M. -A. Teillet, C. S. Le Lievre, and C. Ziller (1982) The neural crest and its developmental analysis in avian chimeric embryos. Trends Neurosci. 3: 39 42.

Mudge, A. W. (1981) Effects of chemical environment on levels of substance $\mathrm{P}$ and somatostatin in cultured sensory neurones. Nature 292: 764-766.

Patterson, P. H. (1978) Environmental determination of autonomic neurolransmitter functions. Annu. Rev. Neurosci. 1: $1-17$.

Patterson, P. H., and L. L. Y. Chun (1977) The induction of acetylcholine synthesis in primary cultures of dissociated rat sympathetic neurons. Dev. Biol. 56: 263-280.

Pickel, V. M., K. K. Sumal, and R. J. Miller (1982) Early prenatal development of substance $\mathrm{P}$ - and enkephalin-containing neurons in the rat. J. Comp. Neurol. 210: 411-431

Raper, J. A., M. J. Bastiani, and C. S. Goodman (1983) Pathfinding by neuronal growth cones in grasshopper embryos. I.
Divergent choices made by growth cones of sibling neurons. J. Neurosci. 3: 20-30.

Rothman, T. P., and M. D. Gershon (1982) Phenotypic expression in the developing murine enteric nervous system. J. Neurosci. 2: 381-393.

Specht, L. A., V. M. Pickel, T. H. Joh, and D. J. Reis (1981) Light microscope localization of tyrosine hydroxylase in prenatal rat brain. 1. Early ontogeny. J. Comp. Neurol. 199: $233-253$.

Sternberger, L. A. (1978) Immunocytochemistry, John Wiley \& Sons, New York.

Sulston, J. E., and H. R. Horvitz (1977) Post-embryonic cell lineages of the nematode, Caenorhabditis elegans. Dev. Biol. 56: $110-156$.

Taghert, P. H., K. J. Kotrla, and C. S. Goodman (1982a) Cell determination of neuroblasts and neurons during grasshopper embryogenesis. Neurosci. Res. Program Bull. 20: 813820.

Taghert, P. H., M. J. Bastiani, R. H. Ho, and C. S. Goodman (1982b) Guidance of pioneer growth cones: Filopodial contacts and coupling revealed with an antibody to Lucifer Yellow. Dev. Biol. 94: 391-399.

Taghert, P. H., C. O. Doe, and C. S. Goodman (1983) Cell regulation and determination during development of neuroblasts and neurones in the grasshopper embryo. Nature 307 : $163-165$. 